\title{
Effect of water-in-oil microemulsions and lamellar liquid crystalline systems on the precorneal tear film of albino New Zealand rabbits
}

\author{
Judy Chan' \\ Gamal M El Maghraby ${ }^{1,2}$ \\ Jennifer P Craig ${ }^{3}$ \\ Raid G Alany' \\ 'Drug Delivery Research Unit \\ (2DRU), School of Pharmacy, The \\ University of Auckland, Auckland, \\ New Zealand; ${ }^{2}$ Department of \\ Pharmaceutics, College of Pharmacy, \\ King Saud University, Riyadh, \\ Saudi Arabia; ${ }^{3}$ Department of \\ Ophthalmology, The University of \\ Auckland, Auckland, New Zealand
}

Correspondence: Raid G Alany Drug Delivery Research Unit (2DRU), School of Pharmacy, The University of Auckland, Auckland, New Zealand Tel +6493737599 (ext: 86967)

Fax +6493677192

Email r.alany@auckland.ac.nz

\begin{abstract}
The aim of this study is to investigate the effect of phase transition water-in-oil (w/o) microemulsions (ME) and liquid crystalline systems (LC) on the precorneal tear film (PCTF). The study used six albino NZ rabbits and monitored the integrity and stability of the PCTF before and after instillation of test formulations. The effects were evaluated by assessment of the PCTF lipid layer using interferometry, tear evaporation rate measurements, and indirect estimation of tear volume. Ocular application of test formulations changed the appearance of the PCTF lipid layer, indicating lipid layer disruption. The recovery time was longer in case of ME compared with an aqueous solution (SOL). The tear evaporation rate was increased after application of both $\mathrm{ME}$ and LC systems compared with the SOL, with the LC system showing the greatest effect. Tear volume measurement results revealed minimal changes associated with the instillation of both $\mathrm{ME}$ systems. Whilst phase transition w/o ME systems can interact with the PCTF lipid layer in albino New Zealand rabbits, their effect on the volume of resident tears was found to be minimal.
\end{abstract}

Keywords: ocular microemulsion; tear film; dry eye

\section{Introduction}

Topical application of drugs to the surface of the eye is the most common treatment modality for numerous ophthalmic disorders. However, only $5 \%$ of the applied drug in conventional eye drops penetrates the cornea/sclera and reaches intraocular tissues with the rest of the instilled dose undergoing transconjunctival systemic absorption or drainage via the nasolacrimal duct before transnasal or gastrointestinal absorption (Meseguer et al 1994; Lang 1995). Accordingly, one of the major challenges facing pharmaceutical formulators is to develop topical ocular delivery systems with improved ocular retention, increased corneal/scleral drug absorption and reduced systemic side effects whilst maintaining safety, simplicity and convenience. Many strategies have been adopted to partially or fully achieve such objective. These included the use of bioadhesive hydrogels (Durrani et al 1995), formulation of temperature or $\mathrm{pH}$-sensitive in situ gel forming systems (Miller et al 1982; Gurny et al 1985), preparation of collagen shields (Unterman et al 1988; Kaufman et al 1994), application of particulate and vesicular drug delivery systems such as nanoparticles, liposomes and niosomes (Fitzgerald, et al 1987; Calvo et al 1997; Vyas et al 1998; Pignatello et al 2002; Aggarwal and Kaur 2005) or employing micellar systems (Pepić et al 2004).

Pseudoternary systems comprising sorbitan mono laurate (Crill 1), polyoxyethylene 20 sorbitan mono-oleate (Crillet 4), ethyl oleate (Crodamol EO) and water can give rise to at least two colloidal microstructures, namely, water-in-oil microemulsions (w/o ME) and lamellar liquid crystalline systems (LC) as well as an oil-in-water coarse emulsion (EM) (Alany et al 2001). These w/o ME are attractive as vehicles for topical ocular drug delivery. They offer several 
advantages such thermodynamic stability, ability to accommodate drugs with low water solubility, ease of application and possible penetration enhancing effect due to their nonionic surfactant content (Lang 1995). Moreover, and upon phase transition of selected w/o ME systems due to aqueous dilution, the resultant LC system were previously shown to display the desirable high viscosity and psuedoplasticity. Such LC systems are viscous enough to promote precorneal retention, yet can thin and display less resistance upon blinking. Such formulations are therefore expected to perform better than traditional viscous formulations (Alany et al 2001). We have previously shown that selected colloidal systems (ME 5\%, ME 10\%, and LC) can prolong the precorneal retention and improve the ocular bioavailability of the model hydrophilic drug, pilocarpine hydrochloride (Alany et al 2006). In addition, we have recently shown that these systems can prolong the release of pilocarpine hydrochloride in vitro with extended duration of action in vivo (Chan et al 2007). Furthermore, we have shown that these surfactant-containing systems were practically nonirritant to the eye according to the results obtained using a modified HET-CAM test (Alany et al 2006). However, it should be emphasized that the HET-CAM is a model for the conjunctiva (Leighton et al 1985). It follows that the previously generated ocular irritation data (Alany et al 2006) did not consider any potential interactions with and destabilizing effects on the precorneal tear film (PCTF) which is one of the principal defense mechanisms of the eye, hence the need to address that.

The PCTF, is a delicate layer that maintains ocular hydration and ensures a smooth optical refracting surface. It lubricates the corneal epithelium and the constant turnover of tears allows for the effective removal of irritants. Furthermore, the tears contain antimicrobial defences, such as immunoglobulins, lysozymes, and white blood cells, which help protects the eye from potential infections (Terry 2001). The PCTF comprises three layers (Figure 1). The innermost part is composed mainly of glycoproteins and is of importance for wetting the corneal and conjuntival epithelia as well as promoting the stability of the watery tear film (Vale and Cox 1985; Terry 2001). The central layer is aqueous in nature and is produced by the main and accessory lacrimal glands; and the outermost layer of the PCTF is a thin lipid layer, produced by 20-30 meibomian glands located in the tarsal plates of the rabbit eye lids. Disruption of this lipid layer leads to exposure and subsequent evaporation of the underlying aqueous tears, causing an evaporative dry eye. This is a potentially serious manifestation associated with debilitating symptoms and without appropriate treatment predisposes the patient to ocular surface damage.

The primary objective of this study was to evaluate the effect of selected colloidal systems (ME 5\%, ME 10\%, and LC) on the PCTF in rabbits. It investigated the effect of these systems on the outer lipid layer of the PCTF with the aqueous solution formulation (SOL) being used as the control. The study was conducted on albino NZ rabbits and monitored three aspects, these are:

- Interferometric assessment of the lipid layer integrity,

- Measurement of the tear evaporation rate and;

- Indirect estimation of tear volume.

\section{Materials and methods}

\section{Materials}

Ethyl oleate (Crodamol EO), sorbitan mono laurate (Crill 1) and polyoxyethylene (20) sorbitan mono-oleate (Crillet 4 super) were obtained form BTB Oleochemicals (Auckland, New Zealand). Pilocarpine hydrochloride (PHCl) USP grade was obtained from Sigma Chemical Co. (St. Louis, USA). Deionised water was used as the aqueous component.

\section{Preparation of ocular formulations}

Table 1 outlines the composition of the tested ocular formulations. The formulations contained the two nonionic surfactants (Crill 1 and Crill 4, blended at 2:3 weight ratio) with ethyl oleate (Crodamol EO) as the oil component. The formulations were prepared at a constant surfactant blend/oil weight ratio (3:2). These formulations were characterized as two W/O ME formulations containing an aqueous component at 5\% and $10 \% \mathrm{w} / \mathrm{w}$ (ME 5\% and ME 10\%), respectively with the later being close to the W/O ME-LC phase boundary. The other formulations were an LC formulation with an aqueous component at 26\% w/w, an O/W EM system with $85 \% \mathrm{w} / \mathrm{w}$ aqueous and an aqueous solution (SOL).

Table I Composition of the investigated formulations

\begin{tabular}{ll}
\hline Formulation & Composition (weight ratios) \\
\hline ME 5\% & PHCl,Water, Crill I, Crillet 4, Crodamol EO (I:4:22.8: \\
& $34.2: 38)$ \\
ME 10\% & PHCl,Water, Crill I, Crillet 4, Crodamol EO (I: 9:2 I.6: \\
& $32.4: 36)$ \\
LC & PHCI,Water, Crill I, Crillet 4, Crodamol EO (I:25: I 7.8: \\
& 26.6:29.6) \\
EM & PHCI,Water, Crill I, Crillet 4, Crodamol EO (I:84:3.6: \\
SOL & $5.4: 6)$ \\
\hline
\end{tabular}

Abbreviations: ME,W/O microemulsion; LC, lamellar liquid crystalline system; EM, $\mathrm{O} / \mathrm{W}$ coarse emulsion; $\mathrm{SOL}$, aqueous solution; $\mathrm{PHCl}$, pilocarpine hydrochloride. 


\section{AIR}

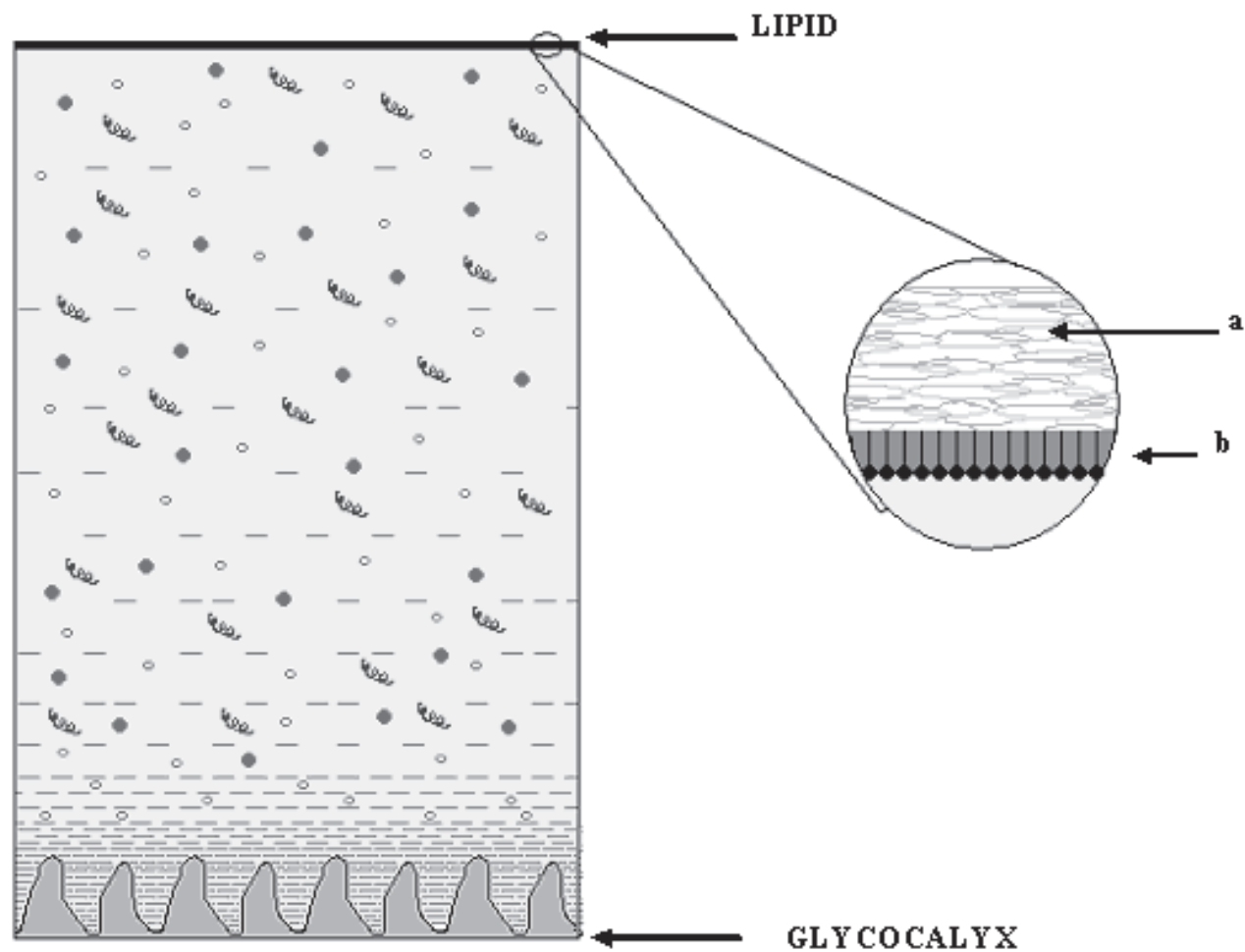

Key:

a Thick non-polar hydrophobic lipids such as wax esters and sterol esters.

b Thin polar lipid phase containing hydrophilic polar lipids such as phospholipids

- Metabolites such as glucose and urea

- Electrolytes such as sodium, potassium, magnesium, calcium, chloride, bicarbonate, phosphate and hydrogen ions.

Proteins including tear lipocalin, albumin, antibodies, lysozyme, beta-lysin, lactoferrin, lactate dehydrogenase, pyruvate kinase, malate dehydrogenase and amylase.

- Soluble mucins

Membrane Mucins

Glycocalyx is a fine layer of glycoproteins covering the ocular surface

Figure I Schematic illustration of the precorneal tear film (PCTF) outlining comprising layers (Not to scale).

The model hydrophilic drug was incorporated by replacing the water content with an aqueous solution containing drug sufficient to produce a constant concentration $(1 \% \mathrm{w} / \mathrm{w})$ in all formulations. The oil, surfactant blend and the aqueous phase were vortex mixed for 10 minutes and the resulting systems were left to equilibrate overnight at ambient temperature before conducting the in vivo evaluation. The detailed formulation method and phase behavior for these systems has been previously described (Chan et al 2007).
The flow properties and the viscosity of the formulations were determined using a DV III cone and plate Brookfield viscometer, fitted with a CP 52 spindle and operated using a Rheocalc programme (Brookfield Engineering Laboratories Inc., Stoughton, MA, USA).

\section{Application of the test formulations}

The studies were conducted using six New Zealand albino rabbits weighing between 2 and $3 \mathrm{~kg}$ each. The rabbits were 
fed a normal diet and exposed to alternating 12 hours light and dark cycles. The rabbits were kept in restraining cages with adequate space for movement throughout the course of the experiment. All experiments were performed in the same room under the same air conditioning and standard lighting conditions; light intensities were monitored using a photometer (Lux Meter Q-1400, DSE). The same rabbits were used to evaluate all formulations with at least 48 hours wash out period between each application. Each test formulation $(20 \mu \mathrm{L})$ was instilled into the lower fornix of the conjunctival sac of the left eye using a positive displacement pipette with the right eye serving as a control.

All animal manipulations were conducted in accordance with the guidelines of The University of Auckland Animal Ethics Committee.

\section{Lipid layer thin film interferometery}

The lipid layer quality and an estimate of lipid layer thickness were determined using a Keeler Tearscope ${ }^{\mathrm{TM}}$ (Keeler Ltd, Berkshire, UK). The Tearscope ${ }^{\mathrm{TM}}$ is equipped with a diffuse cold cathode lamp and is used in conjunction with a hand held nonilluminated biomicroscope (Haag-Streit Bern, Switzerland, 16 $\times$ magnification) to provide an interferometric assessment of the lipid layer. The light generated by the Tearscope ${ }^{\mathrm{TM}}$ is reflected from the surface of the lipid layer and from the interface between that lipid and aqueous layers of the tear film producing various interference patterns (Yokoi and Komuro 2004). Where the lipid layer is approximately $100-140$ nm (approximately $1 / 4$ of the wavelength of visible light), coloured fringes become visible. At thicknesses below this, the colour patterns disappear and there is an apparent loss of lipid layer structure. This apparent loss of structure or so-called "disruption" occurs when a drop of a conventional eye drop or any other ocular dosage forms is instilled into the eye.

Figure 2 shows the appearance of both an intact and disrupted PCTF lipid layer. The lipid layer quality and thickness was assessed immediately before application of the test formulation. This assessment provided the baseline coloured fringe pattern for each rabbit. Immediately after instillation of the test formulation the tear film lipid layer was monitored and the time taken for the lipid layer to resume its baseline pattern was recorded. This time was taken as a measure of the degree of disruption of the PCTF lipid layer.

\section{Lipid layer integrity (Evaporimetry)}

Disruption of the PCTF lipid layer can cause an increased evaporation of the underlying aqueous layer which can lead to an evaporative dry eye. Accordingly, the tear evaporation rate (flux) was taken as an indirect, but objective measure of the stability of the precorneal tear film. These measurements were carried out using a Servomed Evaporimeter, model EP3 (Servomed, Varberg, Sweden). This Evaporimeter has two sensors, located a known distance a part $(1 \mathrm{~cm})$ and held at a known distance $(1.5$ $\mathrm{cm}$ ) from the evaporating surface (the eye) to measure the vapour pressure gradient, which is proportional to the evaporation rate.

Before instillation of the test formulations, three consecutive baseline readings were obtained. The procedure was conducted by placing the probe at a distance of $1.5 \mathrm{~cm}$ from the surface of the eye with a modified swimming goggle mounted to the probe. This modification prevents the probe from touching the ocular surface, which may damage the sensors and irritate the eye. The goggle was placed over the left eye of the rabbit for a period of 120 seconds. After allowing 60 seconds for stabilization, measurements were taken only from 60-120 seconds (Craig and Blades 1997). After establishment of the baseline measurement, $20 \mu \mathrm{l}$ of the test formulation was instilled into the lower conjunctival sac of the rabbit's left eye. The initial evaporation rate was measured one and a half minutes after instillation, and readings were taken every 15 minutes, until three consecutive baseline readings were recorded.

The recorded evaporation rates were plotted as a function of time. The area under the tear evaporation rate versus time curve (AUC) was calculated for the individual formulations using the trapezoidal method.

\section{Tear volume measurements (Phenol Red thread test)}

Indirect tear volume measurements were conducted by inserting a phenol red impregnated thread (Zone-Quick, Menicon Co. Ltd, Nagoya, Japan) into the inferior fornix of the conjunctival sac (Figure 3). The pH sensitive thread soaks up the tear fluid, changes from yellow to red on contact with tears and the wetted length is measured after 15 seconds (Figure 3). Less than $10 \mathrm{~mm}$ of thread wetting in 15 seconds is considered to be indicative of tear deficiency in humans.

To establish baseline tear volume, the thread was placed into the inferior fornix of the conjunctival sac of the left eye of the rabbit for 15 seconds, after which the length of the tear wetted portion was measured (Craig and Blades 1997). The test formulation $(20 \mu 1)$ was then instilled into 
(a)

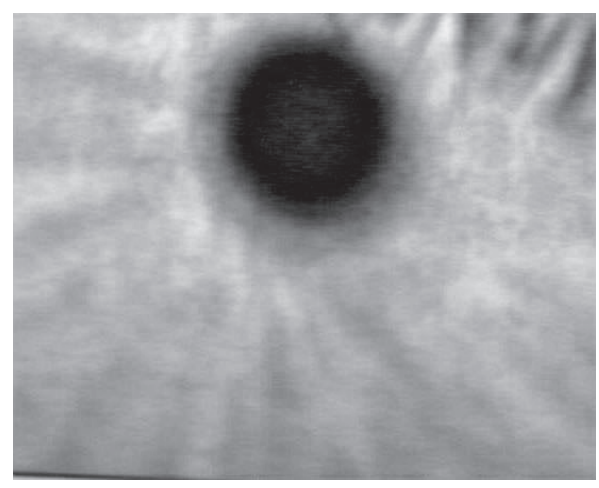

(b)

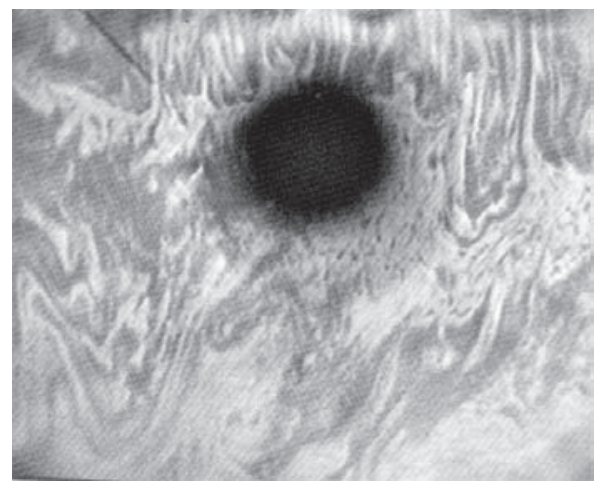

Figure 2 The appearance of the precorneal tear film lipid layer, (a) intact and (b) disrupted.

the lower fornix of the conjunctival sac of the same eye, and a second phenol red thread test was conducted five minutes post-instillation to assess the tear volume changes. The difference between the measured wetted length after and before instillation was recorded and taken as an indirect measure

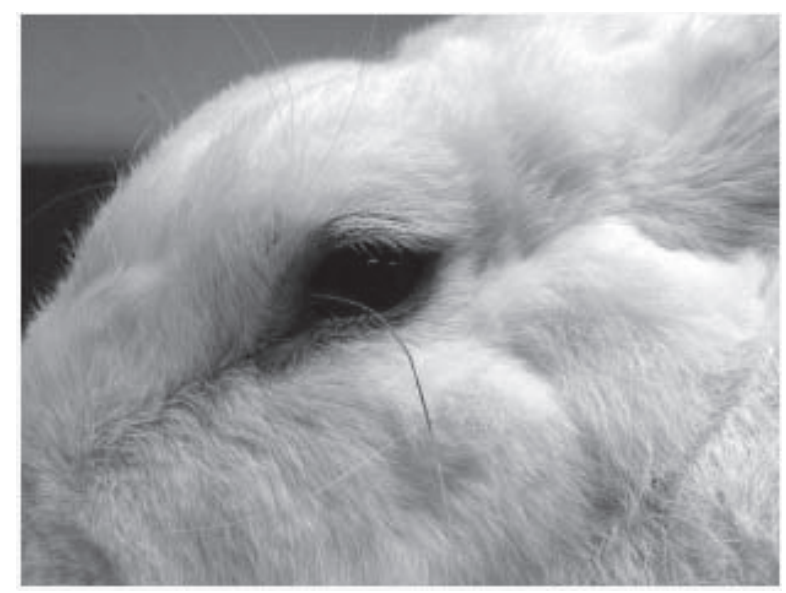

Figure 3 The Phenol Red thread test. Note the dark and bright discoloration of the thread indicating wet and dry portions respectively. for the tear volume. The volume of the resident tears in the precorneal area is directly proportional to the wetting length of the phenol red thread.

\section{Statistical analysis}

A two-way analysis of variance (ANOVA) was used to determine the overall statistically significant difference between the formulations and biological differences between the animals. After excluding any animal effect due to insignificant differences between the six rabbits, a one-way ANOVA followed by Tukey's pairwise comparisons at a $95 \%$ confidence interval was used to test for significant difference between formulations. The two-way, one-way ANOVA and Tukey's pairwise comparisons were performed using Minitab ${ }^{\mathrm{TM}}$ statistical analysis programme Release 12 .

\section{Results}

The quality and stability of the PCTF lipid layer was monitored before and after instillation of the phase transition formulations using the Tearscope ${ }^{\mathrm{TM}}$. As expected, the initial observations conducted (before instillation of the test formulations) showed an intact lipid layer as indicated by the normal coloured fringe patterns (Figure 2a). This was the case with all rabbits. After application of the test formulations, the appearance of the lipid layer changed from its normal (Figure $2 \mathrm{a}$ ) to the disrupted appearance (Figure 2b), indicating a degree of lipid layer perturbation and disruption. To compare the test formulations, the time taken for the lipid layer to return to its baseline level was determined and the results are presented in Table 2. Statistical analysis using the twoway ANOVA revealed no significant differences between rabbits $(\mathrm{p}>0.05)$. On the other hand Tukey's pairwise comparison indicated significant differences between the SOL and all other formulations $(\mathrm{p}<0.05)$ but no significant differences between ME 5\%, ME 10\%, LC and the coarse emulsion EM ( $p>0.05)$. The solution formulation (SOL) showed a significantly faster recovery of the lipid layer to

Table 2 Time required by the lipid layer to return to its baseline pattern as measured by the Tearscope ${ }^{T M}$ after instillation of the test formulations. Results are presented as mean $\pm S D, N=6$

\begin{tabular}{ll}
\hline Formulation & Average recovery time (seconds) \\
\hline ME 5\% & $271.7 \pm 29.3$ \\
ME 10\% & $281.7 \pm 26.5$ \\
LC & $395.0 \pm 41.1$ \\
EM & $255.7 \pm 22.9$ \\
SOL & $117.5 \pm 12.6^{*}$ \\
\hline
\end{tabular}

Note: "Significantly different from all the other formulation $(p<0.05)$. 
its baseline state; while the liquid crystalline system (LC) demonstrated the greatest interaction with the lipid layer with the recovery time period (395 seconds), being longer than all other formulations (Table 2). A similar result was recorded for the two microemulsion systems (ME 5\% and ME $10 \%$ ), with average recovery times of 271.7 seconds and 281.7 seconds, respectively. This was also comparable to the lipid layer recovery time generated by the coarse emulsion (EM), being 255.7 seconds.

The tear evaporation rate (flux) was measured as the second parameter in relation to the potential interaction of these test colloidal systems with the PCTF. The rate of tear evaporation was measured as a function of time after instillation of the test formulations. The results are shown in Figure 4. It is evident that all three colloidal systems (ME 5\%, ME 10\%, and LC) had a pronounced effect on the tear evaporation rate. On the other hand the coarse emulsion EM demonstrated a less pronounced effect, where as SOL exhibited a minimal effect (Figure 4). For objectively comparing the different formulations with respect to their effect on the extent of tear evaporation and the duration of tear evaporation, the area under the tear evaporation rate versus time curve (AUC) was calculated. The results are presented is Table 3 . The liquid crystalline formulation (LC) produced the highest tear evaporation rate as reflected by an AUC of $10.65 \mathrm{~g} . \mathrm{m}^{-2}$, followed by the two microemulsion systems with AUC values of $8.15 \mathrm{~g} . \mathrm{m}^{-2}$ and $6.2 \mathrm{~g} . \mathrm{m}^{-2}$ for ME $5 \%$ and ME $10 \%$, respectively. The coarse emulsion formulation (EM) resulted in an AUC value of $4.05 \mathrm{~g} . \mathrm{m}^{-2}$, whilst the SOL produced a minimal effect on tear evaporation as reflected by the AUC $\left(0.40 \mathrm{~g} . \mathrm{m}^{-2}\right)$. The statistical analysis revealed significant differences $(\mathrm{p}<0.05)$ between both phase transition formulations (ME 5\% and ME 10\%) and the solution formulation (SOL). Furthermore, the LC formulation produced a significantly higher AUC $(p<0.05)$ compared to the EM and SOL formulations but was not significantly different from both ME formulations ( $p>0.05)$.

The volume of tears was indirectly measured as the third parameter reflecting the effect of the test formulations on the stability and integrity of the PCTF lipid layer. This was determined using the Phenol Red impregnated thread. The results were calculated as the difference in the length of the wetted portion of the thread measured after and before instillation of the formulations. The results are presented in Table 4. The largest change was apparent with the LC formulation followed by the coarse emulsion (EM) then the solution (SOL) with both phase-transition microemulsion

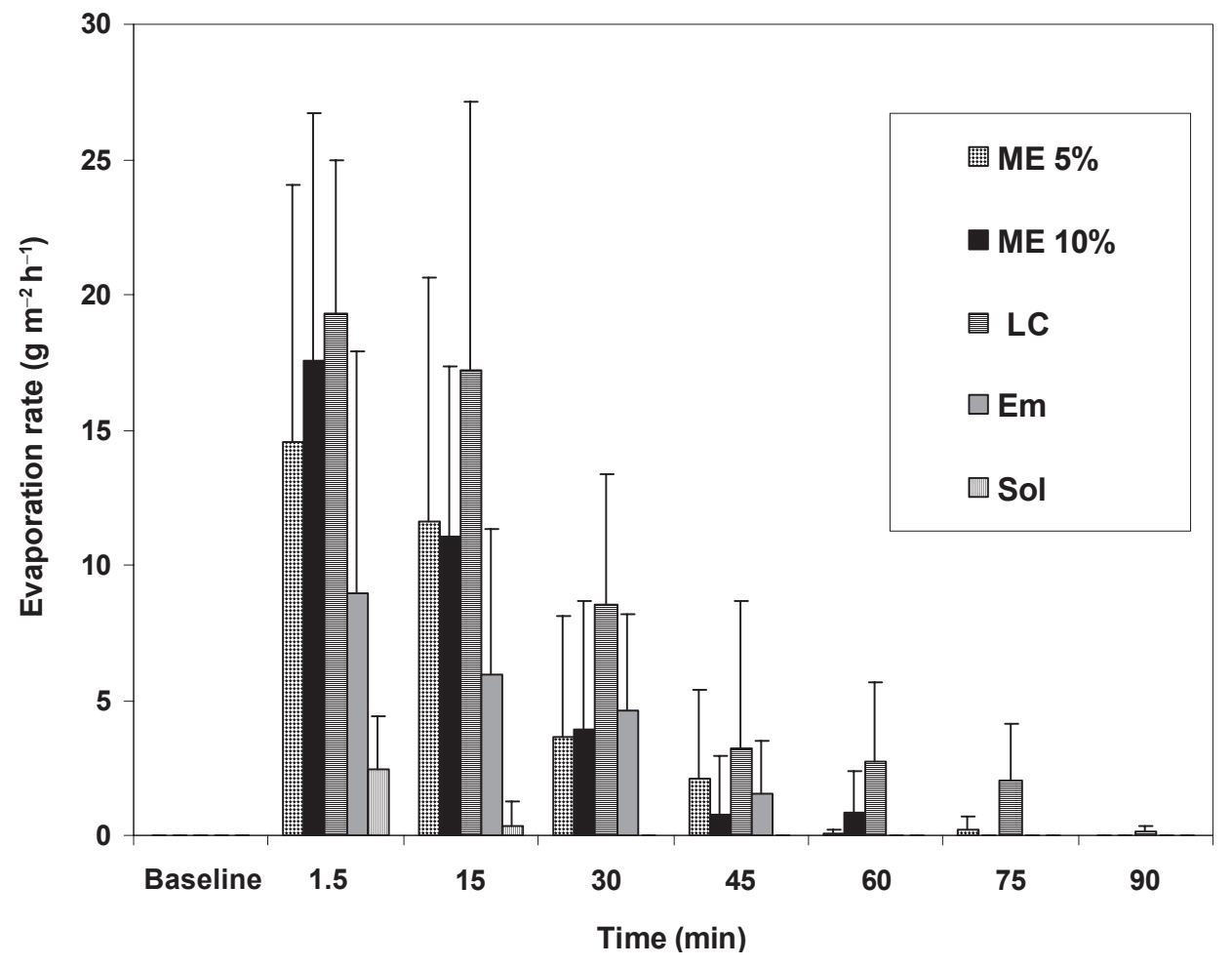

Figure 4 The tear evaporation rate, measured using a Servomed Evaporimeter. Data were recorded at different time points after instillation of different formulations. Results are presented as mean $\pm \mathrm{SD}, \mathrm{N}=6$. 
Table 3 The area under the evaporation rate versus time curve (AUC) presented as mean values $\pm S D, N=6$ in relation to surfactant content and viscosity of the test formulations

\begin{tabular}{lllll}
\hline Formulation & AUC $\left(\mathbf{g ~ m}^{-2}\right)$ & Surfactant content $(\% \mathbf{w} / \mathbf{w})$ & Type of flow & Viscosity $(\mathbf{m P a})$ \\
\hline ME $5 \%$ & $8.15 \pm 1.75^{*}$ & 57.0 & Newtonian & 112 \\
ME 10\% & $6.20 \pm 1.28^{*}$ & 54.0 & Newtonian & 167 \\
LC & $10.65 \pm 2.22^{* *}$ & 44.4 & Non-Newtonian*** & 5487 \\
EM & $4.05 \pm 1.32$ & 9.0 & Newtonian & 4.17 \\
SOL & $0.40 \pm 0.15$ & 0 & Newtonian & 1.1 \\
\hline
\end{tabular}

Notes: "Significantly different from SOL $(p<0.05)$; ${ }^{* * *}$ Significantly different from EM and SOL $(p<0.05)$; ${ }^{* * * *}$ Type of flow is non-Newtonian pseudoplastic.

formulations (ME 5\% and ME 10\%) producing the smallest effect on tear volume as indicated by the smallest difference in the length of the wetted thread (Table 4).

\section{Discussion}

Initial Tearscope ${ }^{\mathrm{TM}}$ examination of the rabbit eye revealed consistent appearance of the PCTF lipid layer for all rabbits. This indicated that the study employed rabbits with healthy eyes. Shortly after instillation of the individual formulations the appearance of the lipid layer began to change from the normal to the disrupted appearance (Figure 2b). Calculating the time needed for the lipid layer to recover its normal appearance, the solution (SOL) required the shortest. On the other hand, the coarse emulsion (EM) as well as both ME formulations required significantly longer time compared with the solution (SOL), with the LC system recording the longest time amongst all tested formulations. The importance of the lipid layer for the stability of the PCTF was previously reported. The major function of the lipid layer is to prevent the evaporation of tears with a disrupted lipid layer being associated with the well-known evaporative dry eye syndrome (Craig and Tomlinson 1997; Johnson and Murphy 2004). Accordingly, the evaporation rate of the tears was monitored before and after instillation of the tested formulation. As with the Tearscope ${ }^{\mathrm{TM}}$ results, the solution (SOL) yielded the lowest evaporation rate, whereas the LC system displayed the highest evaporation rate. It should be noted that the higher rates of evaporation obtained after recovery

Table 4 The difference in the wetted length of the Phenol Red thread calculated from post-instillation minus pre-instillation length. Results presented as mean $\pm S D, N=6$

\begin{tabular}{ll}
\hline Formulation & Average Difference $(\mathbf{m m})$ \\
\hline ME 5\% & $4.67 \pm 2.33$ \\
ME I0\% & $4.17 \pm 2.22$ \\
LC & $11.33 \pm 5.19$ \\
EM & $8.33 \pm 3.89$ \\
SOL & $6.33 \pm 3.47$ \\
\hline
\end{tabular}

of the lipid layer is indeed unexpected. This may indicate a possible increased tear production and/or flow post formulation instillation.

The physicochemical model of the lipid layer of the PCTF has revealed a relatively thick outer layer comprising nonpolar lipids with a thinner polar inner layer comprising phospholipids (McCulley and Shine 1997). Such a structure is most likely to be destabilised by any formulation containing surfactants. Accordingly, the surfactant content of individual formulation was compared with the AUC values (Table 3). The rank of the AUC did not relate with the surfactant content for the individual formulations. According to Table 3, the LC formulation which has an intermediate amount of surfactant produced the highest AUC and evaporation rate. This may indicate that the surfactant concentration is not the main variable affecting the stability of the lipid layer. Previous investigations on these systems revealed similar surface tension values for all the ME formulations and concluded that these formulations are in the reverse micellar state (Chan et al 2007). This indicates that the concentration of the surfactant blend was above the critical micelle concentration in all cases. This explains the more powerful effect of the colloidal formulations compared with the solution formulation (SOL).

Previous rheological investigations of the test formulations revealed a dependence of the viscosity on the water content with the viscosity increasing with increasing the water content, reaching its maximum with LC formulation and dropping back to values close to the minimum in case of the EM (Table 3) (Alany et al 2001). More viscous formulations can promote longer ocular retention which has been previously reported for these formulations (Alany et al 2006; Chan et al 2007). This can explain the more pronounced effect of the LC system on the PCTF lipid layer. The rheological behaviour and viscosity for the formulations under investigation have been previously reported (Alany et al 2001; Chan et al 2007) and are presented in Table 3. 
The Phenol Red thread test was used to indirectly measure the tear volume, with the results being calculated as post-instillation minus pre-instillation wetted length. The test provided unexpected results which did not relate well to those obtained from either the interferometric evaluation or the tear evaporation measurement. Surprisingly both ME systems displayed the least difference on average. It has been previously proposed that the Phenol Red thread test measures uptake of a (small) amount of fluid residing in the eye, stimulates a low degree of reflex tearing, and reflects the absorption characteristics of the thread dependent on the biophysics or composition of tears (Tomlinson et al 2001). Our results can be explained by the tendency of both ME systems to absorb water by passive diffusion from the surrounding environment (tear fluid). This is mainly due to their relatively low water content and the resultant concentration gradient that acts as the driving force for the water mass transfer process from the surrounding environment into the ME formulation. It should be noted that despite the current findings, these formulation were previously reported to be well tolerated by the eye, based on irritation test using the HET-CAM model (Alany et al 2006). However, it should be noted that the HET-CAM model represents the conjunctiva with no direct relation to the PCTF (Leighton et al 1985). It is encouraging to note that none of these systems induced any visible signs of irritation or discomfort when instilled into the eye of albino New Zealand rabbits (Chan et al 2007).

Moreover, it is interesting to report that some undesirable effects have been reported, even for a conventional eye ointment, which is a commonly used formulation to promote prolonged ocular retention. It has been previously reported that due to the disruption of the PCTF-optical interface, an eye ointment produced local side effects such as visual disturbances and foreign body sensation, although in most cases these were self-limited and well tolerated by patients (MacKeen 1980; Frangie 1985; Leighton et al 1985). It has also been reported that all eye ointments and oils interact with and destabilize the PCTF, and as such increase the risks of acute dry eye upon application (Norn and Opauszki 1977). This was further confirmed by the study conducted by Tsubota and colleagues (1999), where they investigated a calcium-containing ointment for the treatment of dry eye. Patients experienced increased blinking rates, tear evaporation and increased tear break up time upon application of the ointment. In another study that compared a hydro-gel and a vitamin A based ointment, results showed that while no significant differences were found in the aspects of ocular burning, eye dryness and itching sensations between the two formulations, the incidence of visual disturbance and adherent eyelashes were much higher in patients using the ointment (Smolle et al 2004).

Conversely, it has been recently shown that the topical ocular application of a lipid emulsion eye drop increases the PCTF lipid layer thickness and benefit patients with deficient lipid layers experiencing dry eye symptoms (Scaffidi and Korb 2007). Furthermore, there is increasing evidence that lipid based colloidal systems such as liposomes are superior to conventional aqueous solutions in reversing the undesirable symptoms associated with dry eye (Peters et al 2003). Systems based on phosphatidylcholine and phosphatidylinositol were shown to initially destabilize the PCTF lipid layer more than aqueous solutions (Peters et al 2003). Nevertheless, a liposome eye spray based on phosphatidylcholine and the cationic surfactant benzyl dimethylstearyl ammonium chloride showed significant improvement in various dry eye clinical parameters compared with an aqueous solution (Lee et al 2004). Feedback from patients revealed that the liposome eye spray resulted in improvement of dry eye symptoms in $72 \%$ of the cases, despite an initial burning sensation post application (Lee et al 2004). This spray is commercially available for dry eye patients under the trade names Clarymist ${ }^{\mathrm{TM}}$ and Tears Again ${ }^{\mathrm{TM}}$.

\section{Conclusion}

Selected phase transition w/o ME systems can interact with and destabilize the PCTF lipid layer in albino New Zealand rabbits. However, their effect on the volume of resident tears was found to be minimal. Although the presence of a surfactant in an ocular formulation can play a major role in producing undesirable effects, the viscosity of the formulation must also be carefully considered as it may be a significant determining factor in many cases. Despite the desirable rheological properties and prolonged ocular retention of phase transition w/o ME systems, there may still be issues around their compatibility with the PCTF that warrants further investigation.

\section{Acknowledgments}

We would like to thank the NZPERF Scheme for their generous financial support. We also wish to acknowledge the technical support offered by, Vicky Chan, Eamon Duffy, Ben Lam, Derek Lau, and Vijay Pillay.

\section{References}

Aggarwal D, Kaur IP. 2005. Improved pharmacodynamics of timolol maleate from a mucoadhesive niosomal ophthalmic drug delivery system. Int J Pharm, 290:155-9. 
Alany RG, Rades T, Nicoll J, et al. 2006. W/O microemulsions for ocular delivery: Evaluation of ocular irritation and precorneal retention. J Control Rel, 111:145-52.

Alany RG, Tucker IG, Davies NM, et al. 2001. Characterizing colloidal structures of pseudoternary phase diagrams formed by oil/water/ amphiphile systems. Drug Dev Ind Pharm, 27:31-8.

Calvo P, Vila-Jato JL, Alonso MJ. 1997. Evaluation of cationic polymercoated nanocapsules as ocular drug carriers. Int J Pharm, 153:41-50.

Chan J, El Maghraby GMM, Craig JP, et al. 2007. Phase transition water in oil microemulsions as ocular drug delivery systems: In vitro and in vivo evaluation. Int J Pharm, 328:65-71.

Craig J, Blades K. 1997. Preocular tear film assessment. Optician, 213:20-7.

Craig JP, Tomlinson A. 1997. Importance of the lipid layer in human tear film stability and evaporation. Optom Vis Sci, 74:8-13.

Durrani AM, Farr SJ, Kellaway IW. 1995. Influence of molecular weight and formulation $\mathrm{pH}$ on the precorneal clearance rate of hyaluronic acid in rabbit eye. Int $J$ Pharm, 118:243-50.

Fitzgerald P, Hadgraft J, Kreuter J, et al. 1987. A gamma scintigraphic evaluation of microparticulate ophthalmic delivery systems: Liposomes and nanoparticles. Int J Pharm, 40:81-4.

Frangie JP. 1995. Clinical pharmacokinetics of various topical ophthalmic delivery systems. Clin Pharmacokinet, 29:130-8.

Gurny R, Boye T, Ibrahim H. 1985. Ocular therapy with nanoparticulate systems for controlled drug delivery. J Control Rel, 2:353-61.

Johnson ME, Murphy PJ. 2004. Changes in the tear film and ocular surface from dry eye syndrome. Prog Retin Eye Res, 23:449-74.

Kaufman HE, Stuinemann TL, Lehman E, et al. 1994. Collagen-based drug delivery and artificial tears. J Ocul Pharmacol, 10:17-27.

Lang JC. 1995. Ocular drug delivery conventional ocular formulations. $A d v$ Drug Deliver Rev, 16:39-43.

Lee S, Dausch S, Maierhofer G, et al. 2004. A new therapy concept for the treatment of dry eye-the usefulness of phospholipid liposomes. Klin Monatsbl Augenheilkd, 221:825-36.

Leighton J, Nassauer J, Tchao R. 1985. The chick embryo in toxicology: an alternative to the rabbit eye. Food and Chemical Toxicology, 23:293-8.

MacKeen DL. 1980. Aqueous formulations and ointments. Int Ophthalmol Clin, 20:79-92.
McCulley JP, Shine WE. 1997. A compositional based model for the tear film lipid layer. Trans Am Ophthalmol Soc, 95:79-88.

Meseguer G, Gurny R, Buri P. 1994. In vivo evaluation of dosage forms: application of gamma scintigraphy to non-enteral routes of administration. J. Drug Target, 2:269-88.

Miller SC, Donovan MD. 1982. Effect of poloxamer 407 gel on the miotic activity of pilocarpine nitrate in rabbits. Int J Pharm, 12:147-52.

Norn MS, Opauszki A. 1977. Effects of ophthalmic vehicles on the stability of the precorneal film. Acta Ophthalmol (Copenh), 55:23-34.

Pepic I, Jalšenjak N, Jalšenjak I. 2004. Micellar solutions of triblock copolymer surfactants with pilocarpine. Int J Pharm, 272:57-64.

Peters K, Dennis GR, Anderton PG, et al. 2003. The Effects of Novel Amphipathic Block Copolymers on Stabilization of the Rat Tear Film. Invest Ophthalmol Vis Sci, 44:5089-94.

Pignatello R, Bucolo C, Ferrara P, et al. 2002. Eudragit RS100 ${ }^{\circledR}$ nanosuspensions for the ophthalmic controlled delivery of ibuprofen. Euro $J$ Pharm Sci, 16:53-61.

Scaffidi CR, Korb DR. 2007. Comparison of the efficacy of two lipid emulsion eyedrops in increasing tear film lipid layer thickness. Eye Contact Lens, 33:38-44.

Smolle M, Keller C, Pinggera G, et al. 2004. Clear hydro-gel, compared to ointment, provides improved eye comfort after brief surgery. Can J Anaesth, 51:126-9.

Terry MA. 2001. Dry eye in the elderly. Drugs Aging, 18:101-7.

Tomlinson A, Blades KJ, Pearce EI. 2001. What does the phenol red thread test actually measure?. Optom Vis Sci, 78:142-6.

Tsubota K, Monden Y, Yagi Y, et al. 1999. New treatment of dry eye: the effect of calcium ointment through eyelid skin delivery. Br J Ophthalmol, 83:767-70.

Unterman SR, Rootman DS, Hill JM, et al. 1988. Collagen shield drug delivery: therapeutic concentrations of tobramycin in the rabbit cornea and aqueous humor. $J$ Cataract refract Surg, 14:500-4.

Vale J, Cox B. 1985. Drugs and the Eye. London: Butterworth and Co. (Publishers) Ltd.

Vyas SP, Mysore N, Jaitley V, et al. 1998. Niosome based controlled ocular delivery of timolol maleate. Pharmazie, 53:466-9.

Yokoi N, Komuro A. 2004. Non-invasive methods of assessing the tear film. Exp Eye Res, 78:399-407. 
